\section{A multicenter total therapy strategy for de novo adult Philadelphia chromosome positive acute lymphoblastic leukemia patients: final results of the GIMEMA LAL1509 protocol}

\author{
Sabina Chiaretti, ${ }^{1}$ Michela Ansuinelli, ${ }^{1}$ Antonella Vitale,${ }^{1}$ Loredana Elia, ${ }^{1}$ Mabel \\ Matarazzo, ${ }^{1}$ Alfonso Piciocchi, ${ }^{2}$ Paola Fazi, ${ }^{2}$ Francesco Di Raimondo, ${ }^{3}$ Lidia \\ Santoro, ${ }^{4}$ Francesco Fabbiano, ${ }^{5}$ Catello Califano, ${ }^{6}$ Giovanni Martinelli, ${ }^{7}$ \\ Francesca Ronco, ${ }^{8}$ Felicetto Ferrara, ${ }^{9}$ Nicola Cascavilla, ${ }^{10}$ Catia Bigazzi, ${ }^{11}$ \\ Alessandra Tedeschi, ${ }^{12}$ Simona Sica, ${ }^{13,14}$ Nicola Di Renzo, ${ }^{15}$ Angela \\ Melpignano, ${ }^{16}$ Germana Beltrami, ${ }^{17}$ Marco Vignetti ${ }^{2}$ and Robin Foà ${ }^{1}$
}

Volume 106(7):1828-1838

\begin{abstract}
${ }^{1}$ Section of Hematology, Department of Translational and Precision Medicine, Sapienza University, Rome; ${ }^{2}$ GIMEMA Data Center, Fondazione GIMEMA, Rome; ${ }^{3}$ Section of Hematology, Department of General Surgery and Medical-Surgical Specialties, University of Catania, Catania; ${ }^{4}$ Struttura Complessa di Ematologia e Trapianto Emopoietico-A.O. S.G.Moscati, Avellino; ${ }^{5}$ Division of Hematology and Bone Marrow Transplantation, Ospedali Riuniti Villa Sofia-Cervello, Palermo; ${ }^{6}$ Onco-Ematologia Ospedale Pagani, Salerno; ' Seragnoli Institute of Hematology, Bologna University School of Medicine, Bologna; ${ }^{8}$ Operative Unit of Hematology, Grande Ospedale Metropolitano "Bianchi-Melacrino-Morelli", Reggio Calabria; 'Division of Hematology and Stem Cell Transplantation Program, AORN Cardarelli Hospital, Naples; ${ }^{10} \mathrm{Casa}$ Sollievo della Sofferenza IRCCS, San Giovanni Rotondo; ${ }^{11}$ Department of Hematology and Stem Cell Transplantation Unit, C.G. Mazzoni Hospital, Ascoli Piceno; ${ }^{12}$ ASST Grande Ospedale Metropolitano Niguarda, Milan; ${ }^{13}$ Fondazione Policlinico Universitario A. Gemelli, Rome; ${ }^{14}$ Università Cattolica del Sacro Cuore, Rome; ${ }^{15}$ Department of Hematology and Stem Cell Transplant, Presidio Ospedaliero Vito Fazzi, Lecce; ${ }^{16} \mathrm{~A}$. Perrino Hospital, Brindisi and ${ }^{17}$ Policlinico San Martino, Genoa, Italy
\end{abstract}

\section{ABSTRACT}

\section{Correspondence:}

SABINA CHIARETTI

chiaretti@bce.uniroma1.it

ROBIN FOȦ

rfoa@bce.uniroma1.it

Received: May 29, 2020.

Accepted: December 24, 2020.

Pre-published: February 4, 2021.

https://doi.org/10.3324/haematol.2020.260935

(C)2021 Ferrata Storti Foundation

Material published in Haematologica is covered by copyright. All rights are reserved to the Ferrata Storti Foundation. Use of published material is allowed under the following terms and conditions:

https://creativecommons.org/licenses/by-nc/4.0/legalcode. Copies of published material are allowed for personal or internal use. Sharing published material for non-commercial purposes is subject to the following conditions:

https://creativecommons.org/licenses/by-nc/4.0/legalcode, sect. 3. Reproducing and sharing published material for commercial purposes is not allowed without permission in writing from the publisher.

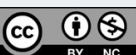

T he GIMEMA LAL1509 protocol, designed for adult ( $\geq 18-60$ years) de novo Philadelphia chromosome positive $(\mathrm{Ph}+)$ acute lymphoblastic leukemia (ALL) patients, was based on dasatinib plus steroids induction - with central nervous system prophylaxis - followed by dasatinib alone in patients in complete molecular response or by chemotherapy and/or allogeneic transplant in patients not reaching complete molecular response. Sixty patients (median age 41.9 years) were enrolled: 33 were p190+, 18 p210+ and nine p190/p210+. At the end of induction (day +85$), 58$ patients $(97 \%$ ) achieved complete hematologic remission. No deaths in induction were recorded. Eleven patients $(18.3 \%)$ obtained complete molecular response. Among the incomplete molecular responders $(n=47), 22$ underwent an allogeneic transplant. Seventeen hematologic relapses occurred (median 7 months; range, 340.1): 13 during consolidation and four post-transplant. ABL1 mutations (five T315I, three V299L, one E281K and one G254E) were found in ten of 13 relapsed cases. With a median follow-up of 57.4 months (range, 4.2-75.6), overall survival and disease-free survival were $56.3 \%$ and $47.2 \%$. A better disease-free survival was observed in patients who obtained a molecular response at day +85 compared to cases who did not. The presence of additional copy number aberrations - IKZF1 plus $C D K N 2 A / B$ and/or PAX5 deletions - was the most important unfavorable prognostic factor on overall and disease-free survival $(P=0.005$ and $P=0.0008)$. This study shows that in adult $\mathrm{Ph}+$ ALL long-term survivals can be achieved with a total-therapy strategy based on a chemotherapyfree induction and, in complete molecular responders, also without further systemic chemotherapy. Finally, the screening of additional copy number aberrations should be included in the diagnostic work-up (clinicatrial gov. Identifier: EudraCT 2010-019119-39). 


\section{Introduction}

Philadelphia positive $(\mathrm{Ph}+)$ acute lymphoblastic leukemia (ALL) was historically recognized as the ALL subset with the most unfavorable outcome. The $\mathrm{Ph}$ chromosome designates the shortened chromosome 22 which encodes the $\mathrm{BCR}-\mathrm{ABL}$ fusion gene/protein kinase. It arises from a translocation termed $\mathrm{t}(9 ; 22)(\mathrm{q} 34 ; \mathrm{q} 11)$ and leads to $B C R$ $A B L 1$ rearrangement. ${ }^{1}$ The incidence of the $\mathrm{Ph}+\mathrm{ALL}$ increases with age, is detected in more than $50 \%$ of elderly B-lineage ALL patients, and represents the most common genetic abnormality in adult ALL. ${ }^{2 \cdot 4}$

The advent of tyrosine kinase inhibitors (TKI) has dramatically changed the management and the prognosis of this high-risk group of patients. ${ }^{5.9}$ In fact, in the pre-TKI era, treatment with standard chemotherapy rarely produced sustained complete remissions and less than $20 \%$ of $\mathrm{Ph}+$ ALL patients were long-term survivors. Allogeneic stem cell transplant (allo-SCT), when feasible according to age and comorbidities, represented the only possibility of cure, if complete hematologic remission (CHR) was achieved..$^{10-12}$ Nowadays, TKI with ${ }^{5,9,13-15}$ or without ${ }^{7,8,16,17}$ systemic chemotherapy in induction represent the gold standard approach. TKI-based schemes have led to a significant improvement in terms of CHR, reached in virtually all patients, disease-free survival (DFS) and overall survival (OS) rates. Nevertheless, the combination of TKI with conventional chemotherapy is aggravated by toxicities and treatment-related mortalities and a $2-7 \%$ death rate during induction has been reported in different studies., ${ }^{9,1318}$ Deintensified chemotherapy regimens have been utilized to limit toxicity associated with the combined use of a TKI and standard chemotherapy. ${ }^{19,20}$

In order to overcome the treatment-related toxicity of the combined TKI-chemotherapy approach, the GIMEMA (Gruppo Italiano Malattie EMatolologiche dell'Adulto) group has applied - over the past 15 years - a chemotehrapy-free induction strategy using first, second or third generation TKI in combination with steroids and central nervous system (CNS) prophylaxis. The first trial with imatinib (clinicaltrials gov. Identifier: LAL0201) was designed for elderly patients (>60 years) and represented the proof of principle that $\mathrm{CHR}$ could be achieved without the use of systemic chemotherapy in Ph+ ALL. In the following study (clinicaltrials gov. Identifier: LAL0904) the same induction backbone was applied to adults up to the age of 60 years of age and patients received chemotherapy as consolidation treatment; if feasible by donor availability and clinical fitness, patients then underwent allo-SCT. The results of this study showed an OS and DFS at 60 months of $48.8 \%$ and $45.8 \%$, respectively. ${ }^{17}$ The subsequent GIMEMA trial (clinicaltrials gov. Identifier: LAL1205) used the second generation TKI dasatinib for 12 weeks as firstline induction treatment for all $\mathrm{Ph}+\mathrm{ALL}$ over 18 years of age and with no upper age limit. All 53 patients obtained CHR at the end of the induction with eight patients $(15.1 \%)$ being in complete molecular response (CMR) and no deaths or progressions were recorded during induction; consolidation was left to each treating center. At 20 months, the OS was $69.2 \%$ and the DFS was $51.1 \%{ }^{16}$ In the subsequent GIMEMA LAL1509 protocol the same induction strategy was utilized - dasatinib in combination with steroids together with CNS prophylaxis - followed by a consolidation strategy modulated according to the molecular response upon induction: patients in CMR continued dasatinib until relapse/progression, while patients who did not reach CMR status continued with systemic chemotherapy and/or allo-SCT. We herein report the final results of the study.

\section{Methods}

\section{Study design, therapy and endpoint}

The GIMEMA LAL1509 trial (clinicaltrials gov. Identifier: EudraCT 2010-019119-39) was designed for adult Ph+ ALL patients (18-60 years). The identification of the $B C R-A B L 1$ transcript was performed centrally within the steroid pre-phase (see below). Induction consisted of 12 weeks of dasatinib administration (140 mg/day), preceded by a 7-day steroid pre-phase (escalating doses from 20 up to $60 \mathrm{mg} / \mathrm{m}^{2}$ ): steroids were administered for 24 days, then tapered until day 31. Patients continued treatment with dasatinib until day 84 after induction, cases in CMR continued dasatinib until progression or minimal residual disease (MRD) increase or occurrence of toxicity (grade $\geq 3$ ). MRD-positive patients were stratified according to transplant eligibility: i) those with a promptly available human leukocyte antigen-compatible donor proceeded to an allo-SCT (by protocol guidelines, a haploidentical donor was not permitted); ii) those with a compatible, but not readily available donor, received one chemotherapy consolidation cycle prior to allo-SCT; iii) those non-eligible for alloSCT received two chemotherapy consolidation cycles. The first consolidation cycle consisted of clofarabine $\left(40 \mathrm{mg} / \mathrm{m}^{2}\right.$, days 1-5) and cyclophosphamide (400 mg/m $\mathrm{m}^{2}$, days 1-5); the second consolidation cycle consisted of 3 days of the same combination; dasatinib was interrupted during the 5 and 3 days of chemotherapy consolidation to avoid excessive toxicity and was restarted as soon as possible, at hematologic recovery. Upon consolidation, patients received dasatinib, until intolerance or relapse. Figure 1 summarizes the treatment scheme and patients' disposition.

CNS prophylaxis is detailed in the Online Supplementary Materials and Methods.

The primary endpoint of the study was to evaluate the rate of patients alive in CHR.

The study was approved by the Ethics Committees of all participating centers; patients gave their written informed consent in accordance with the Declaration of Helsinki.

\section{Hematologic response assessment}

CHR was defined as bone marrow (BM) containing less than $5 \%$ blasts, absence of blasts in the peripheral blood (PB) and no extramedullary involvement. Hematologic relapse was defined as the re-appearance of blasts in the BM and/or in extramedullary sites.

\section{Molecular diagnosis, definition of molecular response and MRD monitoring}

Molecular analyses were centrally performed at the Hematology Center of the Sapienza University of Rome. Molecular testing was carried out to identify the presence of $B C R-A B L 1$ gene in BM samples at baseline and for MRD monitoring. MRD monitoring was performed by quantitative real-time polymerase chain reacxtion ${ }^{21,22}$ during induction at days $+22,+45,+57$ and +85 (end of induction) and at fixed time points according to the post-induction therapy (Online Supplementary Materials and Methods). CMR was defined as a $B C R-A B L 1 / A B L 1=0$, with a confirmatory molecular BM aspirate after 15 days. Molecular relapse was defined as an at least 2-log increase of the $B C R-A B L 1 / A B L 1$ gene expression confirmed in an additional control, which was solicited as soon as a molecular increase was documented. 


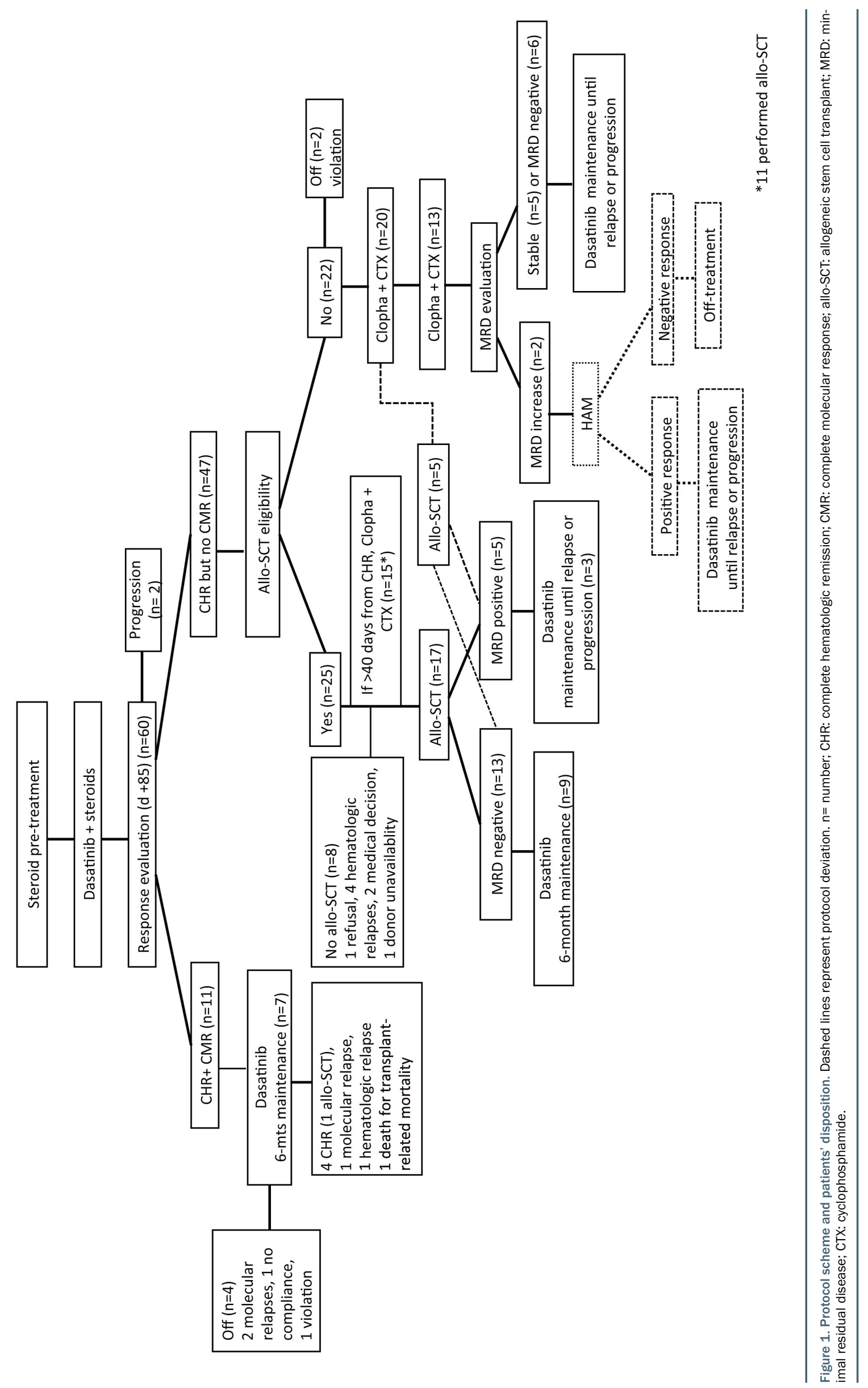


Mutational screening was performed in relapsed cases depending on material availability by Sanger sequencing (Online Supplementary Materials and Methods).

Copy number aberrations analysis was performed on diagnostic samples by Cytoscan arrays, as previously described. ${ }^{23}$

\section{Statistical analysis}

Survival was estimated using the Kaplan-Meier Product Limit. Differences in OS and DFS were evaluated by means of the LogRank test in univariate analysis and by the Cox regression model in multivariate analysis, after assessment of proportionality of hazards. The role of allo-SCT on DFS was evaluated in the Cox regression model by means of a time-dependent covariate. All analyses were performed using the SAS system software (version 9.4). All tests were two-sided, accepting $P \leq 0.05$ as statistically significant. Study data were collected and managed using REDCap ${ }^{24,25}$ electronic data capture tools hosted at the GIMEMA Foundation.

\section{Results}

\section{Patients}

From July 2011 to October 2013, 60 patients with de novo Ph+ ALL were enrolled in the GIMEMA LAL1509 trial. Thirty-four patients were males and 26 females. Median age at presentation was 41.9 years (range, 18.7-59.1). The median white blood cell count (WBC) at diagnosis was $12.4 \times 10^{9} / \mathrm{L}$ (range, 1.4-178). A $B C R-A B L 1$ p190 fusion transcript was detected in 33 patients (55\%), a BCR-ABL1 p210 fusion transcript in 18 (30\%), while 9 patients (15\%) were positive for both transcripts; p210 and p190/p210 were grouped together for all statistical analyses. The median follow-up was 57.4 months (range, 4.2-75.6) (Table 1).

\section{Hematologic response during and after induction}

After the steroid pre-phase, 38 of 60 patients $(63 \%)$ showed a PB blast reduction of $\geq 75 \%$. All patients achieved CHR by day $+57,51$ patients $(85 \%)$ by day +22 . By the end of the induction (day +85$)$, all 60 patients were evaluable for response: 58 patients $(97 \%)$ were in $\mathrm{CHR}$, while two patients showed a disease progression between day +57 and day +85 , both of these were p210-positive.

\section{Minimal residual disease monitoring during and at the end of induction}

Molecular analysis showed that transcript levels rapidly and constantly decreased during the induction phase. The rate of transcript reduction was highly significant $(P<0.0001)$ between the onset of the disease and day +22 , between days +22 and +45 , and between days +57 and +85 . Importantly, a significantly greater clearance was observed in BCR-ABL1 p190 ( $\mathrm{n}=33$ ) versus $\mathrm{p} 190 / 210$ and p210 $(n=27)$ cases at every time point of MRD evaluation $(P=0.0032, P=0.0031, P=0.0016, P=0.007$ at days $+22,+45$, +57 and +85 , respectively) (Figure $2 \mathrm{~A}$ ).

At the end of the induction, CMR was obtained in 11 patients $(18.3 \%)$. In these patients, the rate of $B C R-A B L 1$ transcript clearance was significantly higher at every point of $\mathrm{MRD}$ evaluation since day +22 (Figure $2 \mathrm{~B}$ ).

\section{Post-remission treatment and response}

Figure 1 summarizes the patients' disposition. Of the 11 patients who were in CMR at the end of induction, seven continued with dasatinib: four remain in CHR and CMR after 64, 54, 55 and 53 months (one patient was allografted
Table 1. Patients' characteristics

\begin{tabular}{lc}
\hline Patients' characteristics & $\mathbb{N}=60$ \\
Median age at diagnosis (range) & 41.9 years $(18.7-59.1)$ \\
Sex & \\
M & $34(56.6 \%)$ \\
F & $26(43.3 \%)$ \\
\hline Median WBC count at diagnosis (range) & $12.4 \times 10^{9} / \mathrm{L}(1.4-178)$ \\
Type of fusion transcript & \\
p190 & $33(55 \%)$ \\
p210* & $18(30 \%)$ \\
p190/p210* & $9(16 \%)$ \\
\hline
\end{tabular}

Median FU (range)

57.4 months (4.2-75.6)

*Considered together for subsequent analyses: M: male; F: female; WBC: white blood cell count; FU: follow-up.

after 6 months of dasatinib administration), one experienced a hematologic relapse, one experienced a molecular recurrence and one patient died of transplant-related complications, performed after 6 months of dasatinib maintenance while in CHR and CMR. The remaining four patients stopped dasatinib maintenance: two due to a molecular relapse (BCR-ABL1 T315I mutation was detected in one) and were both allografted, and one patients for a protocol violation due to a medical decision (i.e., transplant allocation within 6 months); all three patients are in CHR after 57, 58 and 60 months. Finally, one patient went off-study for non-compliance.

The 47 patients who were in CHR but not in CMR at the end of induction were evaluated for allo-SCT eligibility. According to protocol guidelines, 25 of 47 patients were considered eligible for the procedure. Seventeen patients were transplanted, 11 after a consolidation cycle with clofarabine plus cyclophosphamide and of these, six patients obtained CMR. Eight patients did not undergo an allo-SCT due to: i) one case of patient's refusal, ii) a documented hematologic or extramedullary relapse in four cases, iii) an unforeseen unavailability of the donor in one case and iv) a medical decision in two cases (one major protocol violation). The remaining 22 of 47 patients were considered ineligible for transplant: 20 patients underwent at least one cycle of clofarabine-cyclophosphamide and 13 received both cycles, with six patients obtaining CMR, while two patients went off protocol prior to consolidation treatment due to a major violation. After the second cycle of clofarabine-cyclophosphamide, 11 of 13 patients started maintenance with dasatinib. Five patients, initially deemed ineligible for transplant, could undergo the procedure after one cycle of consolidation chemotherapy: in two cases for a medical decision, in one case because of the presence of the $B C R-A B L 1$ T315I mutation and in two cases for dasatinib interruption (hematologic toxicity in one and patient's decision in the other). Overall, 22 patients underwent an alloSCT and after the procedure 12 patients restarted dasatinib as maintenance treatment. After allo-SCT, five patients were persistently MRD-positive and two of them went off treatment due to the emergence of the BCR-ABL1 T315I mutation and for a medical decision, respectively, whereas the others restarted treatment with dasatinib. Thirteen patients were MRD-negative after the transplant and in nine patients dasatinib was restarted: i) in three patients dasatinib was administered for the planned 6 months; ii) in one patient for 5 months; iii) in one patient for 3 months; iv) in two patients for 2 and 1 months each. 

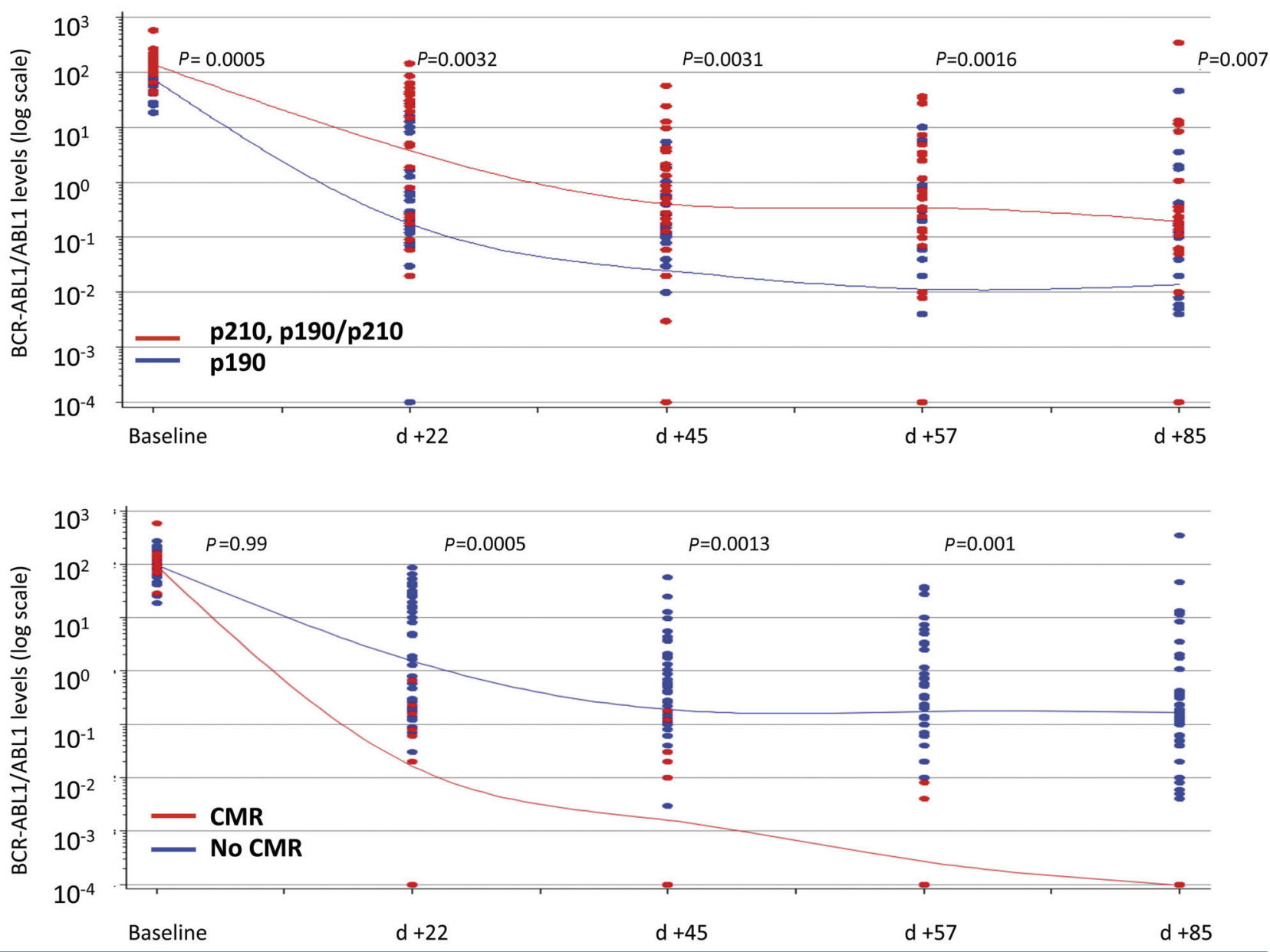

Figure 2. Minimal residual disease clearance. Patients were stratified according to fusion protein (A) and molecular status at the end of induction (B). CMR: complete molecular response; $d=$ day.

\section{Relapse and cumulative incidence of relapse}

Seventeen relapses occurred at a median time of 7 months from the end of induction (range, 3-40.1), of which two were extramedullary (one CNS and one psoas muscle): 13 patients relapsed during consolidation and four patients relapsed after allo-SCT.

At a median follow up of 57.4 months, the cumulative incidence of hematologic relapse (CIR) was 29.8\% (95\% Confidence Interval [CI]: 18.4-42.1) (Figure 3). The CIR, stratified according to the molecular response at day +85 , was $10 \%$ in CMR patients as opposed to $35.6 \%$ in nonCMR patients; this difference did not reach statistical significance, probably due to the small number of relapses.

\section{Toxicity and deaths}

Overall, 188 adverse events (AE) (grade $>2$ ) were recorded in 41 patients (Online Supplementary Table S1), the most frequent were hematologic (124 of $177,66 \%$ ), gastrointestinal (12 of $188,6 \%$ ) and infectious (12 of $188,6 \%$ ). As expected, hematologic toxicity was mainly recorded during induction and was most likely sustained by the disease itself. The most frequent $\mathrm{AE}$ reported with dasatinib was one registered case of pleural effusion and one registered case of peripheral edema, while no cases of pulmonary hypertension were recorded.

Eighteen severe adverse events (SAE) were recorded in ten patients (Table 2), and six SAE were due to treatment: infections and gastrointestinal disorders were the most frequent $(27 \%$ and $22 \%$, respectively).

While no deaths occurred during the induction phase, 24 deaths were recorded in the post-induction phase: 12 for disease progression, 11 in CHR for toxicity and one for both. Causes of death in CHR patients were represented by sepsis in four patients, multi-organ failure (kidney and lung) in two patients and transplant-related mortality in five patients (one for graft versus host disease, two for viral pneumonitis, two for an encephalitis and two for hemolytic anemia).

\section{BCR-ABL1 mutations and copy number evolution analysis}

$B C R-A B L 1$ mutational screening was performed in cases with biological material available at either MRD increase or overt hematologic relapse. A total of 13 cases were screened (ten with a hematologic relapse and three with MRD increase) and the following BCR-ABL1 mutations were detected in ten cases: five cases of T315I, three cases of V299L (one of which with concomitant F317I and F317L mutations), one case of E281K and one case of G254E. No difference in the incidence of $B C R-A B L 1$ mutations was found between p190+ and p210+ patients. Notably, in the three cases with a MRD increase a mutation was detected (E281K, T315I and V299L), but it did not translate into a subsequent hematologic relapse. A total of nine mutated cases were retrospectively evaluated on the diagnostic material and no mutations were detected. 


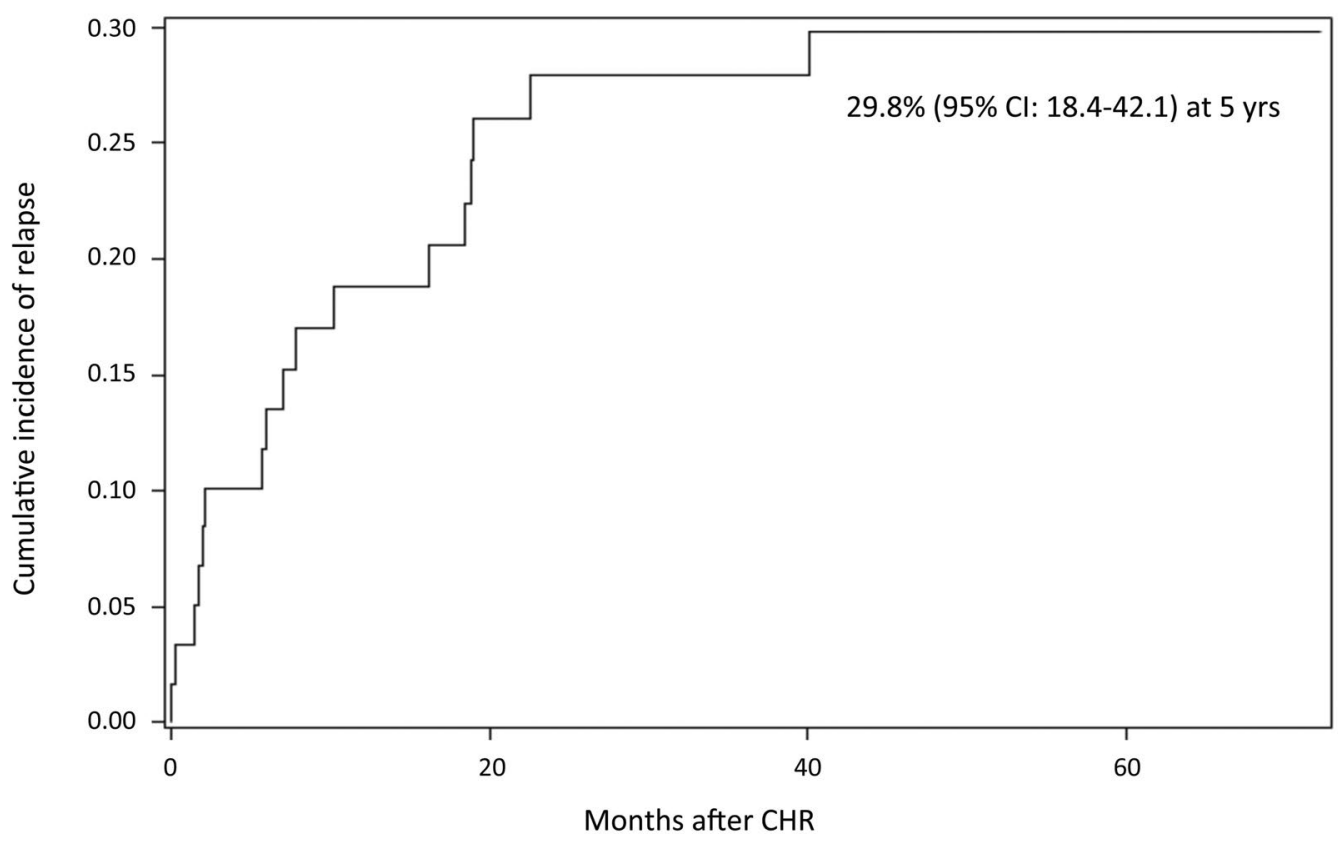

Figure 3. Cumulative incidence of relapse. Cl: Confidence Interval; CHR: complete hematologic remission; yrs: years.

Single nucleotide polymorphism array analysis was carried out at presentation in 39 cases; the most frequent aberrations were deletions of IKZF1 (84.6\%), PAX5 (38.5\%), CDKN2A/B (33.3\%), MLLT3 (33.3\%), JAK2 (28.2\%) and RB1 $(28.2 \%)$. Furthermore, in a small fraction of patients (four of $37 ; 10.8 \%$ ) a deletion involving $M E F 2 C$ was identified.

\section{Disease-free survival and overall survival}

At a median follow up of 57.4 months, the DFS was $47.2 \%$ (95\% CI: 35.6.0-72.6) (Figure 4A), with a median DFS of 30.4 months, and the OS was $56.3 \%$ (95\% CI: 44.5-71.3), with a median $O S$ not reached (Figure 4B). A better, though not significant, DFS was observed in patients who obtained a CMR at day +85 , compared to cases with a MRD positivity $(70 \%$ vs. $42 \%, P=0.08)$ (Figure $5 \mathrm{~A})$. The observed DFS prolongation in CMR patients did not translate into a statistically significant advantage in OS, even though better survival was observed $(70 \%$ vs. $52.9 \%, P=0.27$ ) (Figure $5 \mathrm{~B}$ ). This is probably related to the number of patients and because of the efficacy of consolidation treatments in inducing MRD negativity. DFS was also evaluated on the basis of the fusion protein and the presence of additional copy number evolution (CNA). There were no significant differences in DFS between $B C R-A B L 1$ p190+ patients compared to p210+ and/or p190/p210+ cases (49.7\% vs. 41.7\%, $P=0.4$ ). Likewise, OS was superimposable among the two subsets (56\% vs. $44.2 \%, P=0.9$, not shown).

At variance, the presence of additional CNA cases with the co-occurrence of $I K Z F 1$ plus $C D K N 2 A / B$ and/or PAX 5 (IKZF1 plus) at presentation had a strong impact on DFS and OS had a significantly worse DFS and OS than cases with no alterations and/or with IKZF1 deletions only: $0 \%$ versus $60 \%(P=0.0008)$ and $20 \%$ versus $69.5 \%(P=0.0068)$ at 60 months (Figure 6). Importantly, the presence of IKZF1 deletions alone did not affect DFS. Unfortunately, as also reported by Pfeifer and colleagues, ${ }^{26}$ the negative prognostic impact was also not abrogated when adjusted by transplant
Table 2. Number and types of serious adverse events occurred during the protocol.

\begin{tabular}{lcc} 
Serious adverse events & $\begin{array}{c}\text { Total number } \\
\text { of events }(\mathrm{N}=8)\end{array}$ & $\begin{array}{c}\text { Events related } \\
\text { to dasatinitb or } \\
\text { chemotherapy }\end{array}$ \\
Gastrointestinal system & 4 & 2 \\
Nervous system & 2 & 1 \\
\hline Serous effusions & 2 & 2 \\
Infectious diseases & 5 & 0 \\
\hline Cardiologic toxicity & 1 & 0 \\
General disorders & 3 & 1 \\
\hline Graft versus host disease & 1 & 0 \\
\hline
\end{tabular}

allocation. In addition, patients with $I K Z F 1$ plus $C D K N 2 A / B$ or $P A X 5$ deletions showed a significantly increased CIR $(P=0.0031)$ i.e., all patients harboring these alterations relapsed within 24 months from induction therapy. Patients carrying a $M E F 2 C$ deletion appeared to have a better DFS as opposed to patients without it $(80 \%$ vs. $39.6 \%, P=0.12) .{ }^{23}$ Finally, allo-SCT, evaluated in a Cox model by a time-dependent covariate, did not impact on OS and DFS survival.

\section{Univariate and multivariate survival analyses}

Several factors (age, sex, WBC count, type of fusion protein, additional genetic lesions, etc.) were investigated in univariate analysis to establish their impact on DFS: patients who achieved a CMR after induction, showed a better, though non-significant, DFS, also in multivariate analysis $(P=0.07)$. The co-occurrence of IKZF1 deletions plus $C D K N 2 A / B$ or $P A X 5$ deletions was the only significant factor associated with a worse DFS $(P=0.0008)$ in our cohort of patients. Along the same line, IKZF1 deletions plus $C D K N 2 A / B$ or $P A X 5$ were the only significant factors impacting on $O S$ in both univariate $(P=0.01)$ and multivariate analysis $(P=0.005)$. 
A

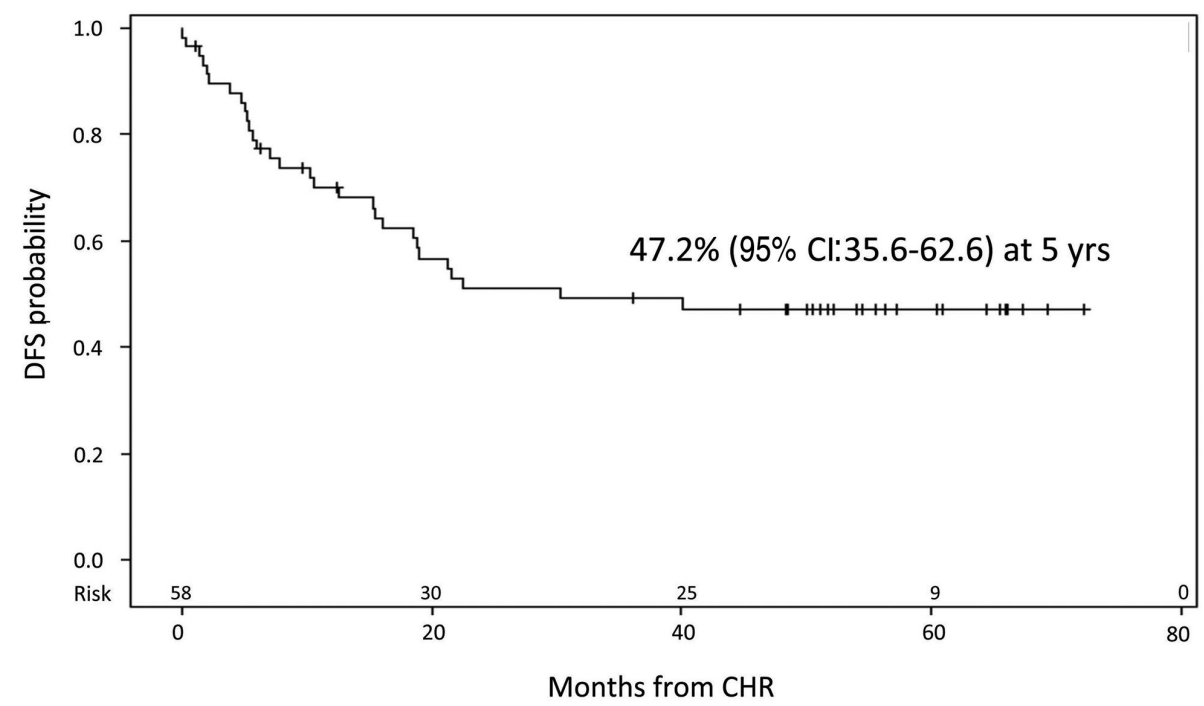

B

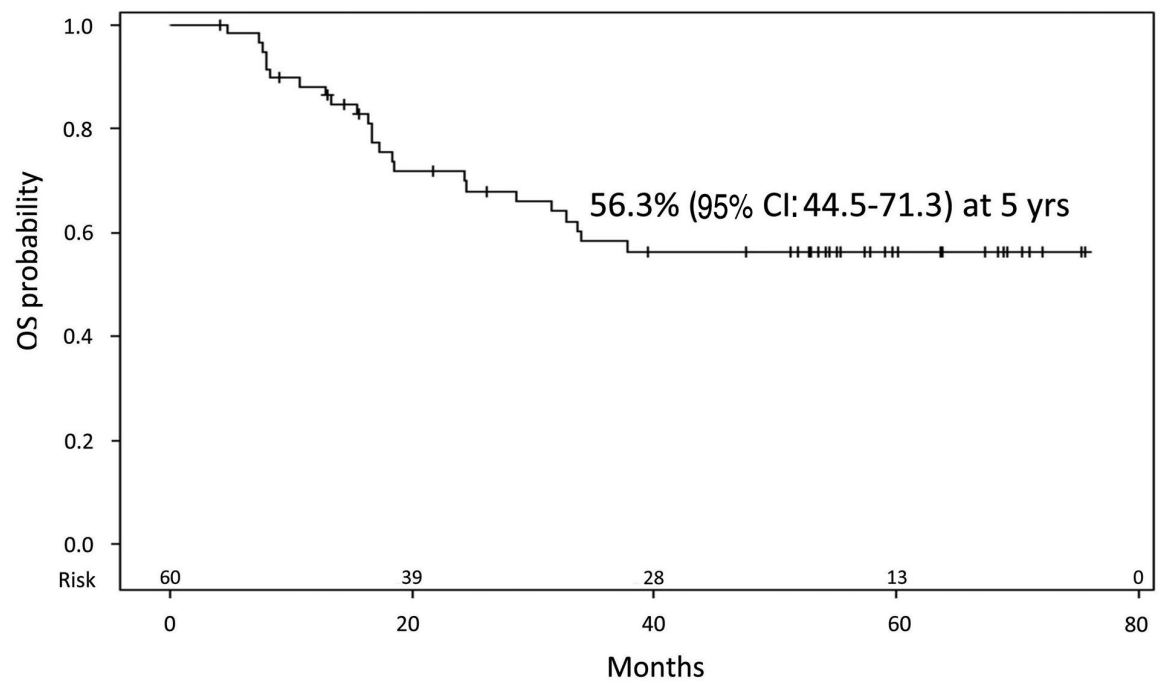

Figure 4. Survival estimates. (A) Disease-free survival (DFS) and (B) overall survival (OS). $\mathrm{Cl}$ : Confidence Interval; CHR: complete hematologic remission; yrs: years.

\section{Discussion}

We herein reported the final results of the GIMEMA LAL1509 trial for adult Ph+ ALL patients. This protocol was based on a total therapy strategy that comprised a chemotherapy-free induction based on dasatinib, plus steroids and CNS prophylaxis, followed by a post-remission treatment tailored according the molecular response. Patients in sustained CMR continued with dasatinib, while patients in CHR who did not achieve CMR underwent chemotherapy consolidation with clofarabinecyclophosphamide plus an allo-SCT for eligible patients. The protocol was designed to evaluate the efficacy of a post-induction treatment modulated on the basis of the depth of molecular response and represents, so far, the first protocol to investigate the possibility of chemotherapy-free consolidation in adult patients (18-60 years) in CMR.

This study confirms the effectiveness of chemotherapy-free induction with dasatinib plus steroids in Ph+ ALL adult patients in inducing a rapid clearance of a neoplastic clone $^{16}$ and also confirms that this strategy is less toxic than combinational strategies, ${ }^{19,27-30}$ since no deaths during induction were recorded, as already reported in previous GIMEMA trials for Ph+ ALL. $7,16,17$

Of the 60 eligible patients, $97 \%$ reached CHR at the end of the induction with two patients in CHR at day +57 , both harboring the p210 fusion protein, who relapsed between day +57 and +85 of the induction phase. At the end of the induction (day +85), CMR - confirmed by a second BM aspirate after 15 days - was achieved in 11 cases (18.3\%). Seven of these 11 patients continued treatment with dasatinib: four patients are still alive and disease-free (one underwent allo-SCT while in CMR), one patient underwent alloSCT and died of transplant-related complications, one patient experienced a hematologic relapse and one patient suffered molecular recurrence; the latter two cases both had an IKZF1-plus phenotype. The remaining four CMR patients were rapidly switched to different therapeutic options, two due to a molecular increase and two due to protocol violations (i.e., transplant allocation and non-compliance). With regards to the two cases with molecular recurrence, a strict $\mathrm{MRD}$ monitoring prevented an overt hematologic relapse (resulting in a benefit for patients) and may thus represent a bias of the study; nevertheless, it further highlights the importance of molecular monitoring, 
A

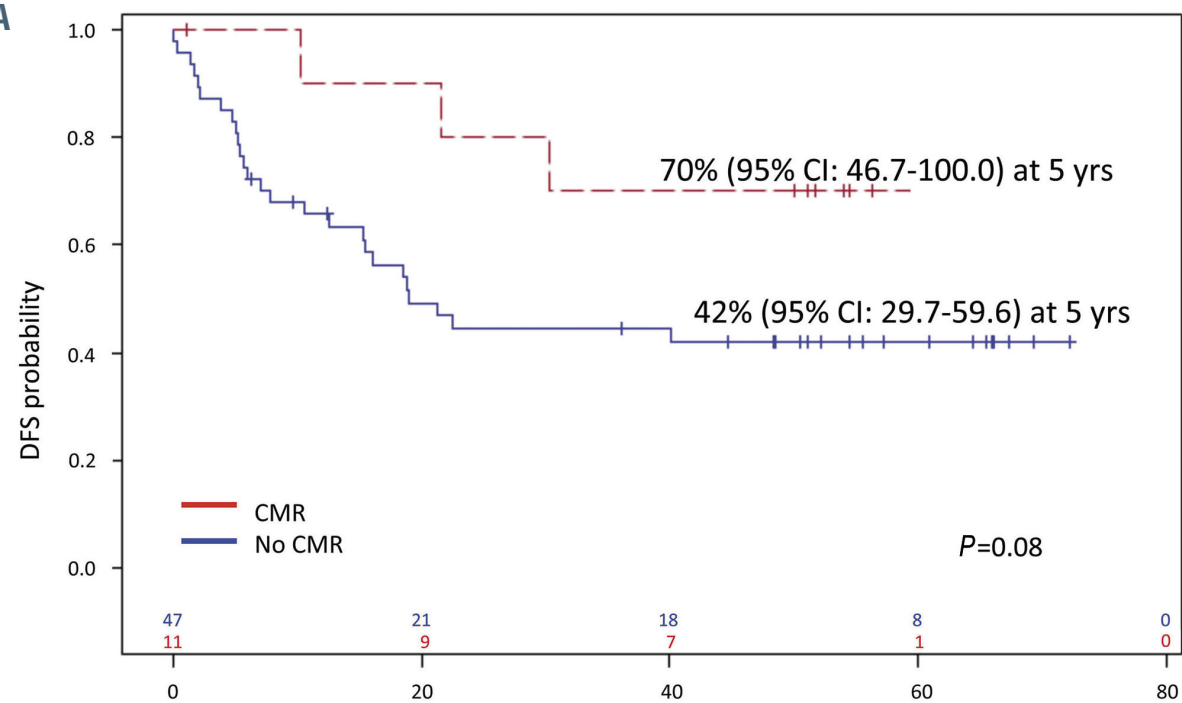

Months from CHR

B

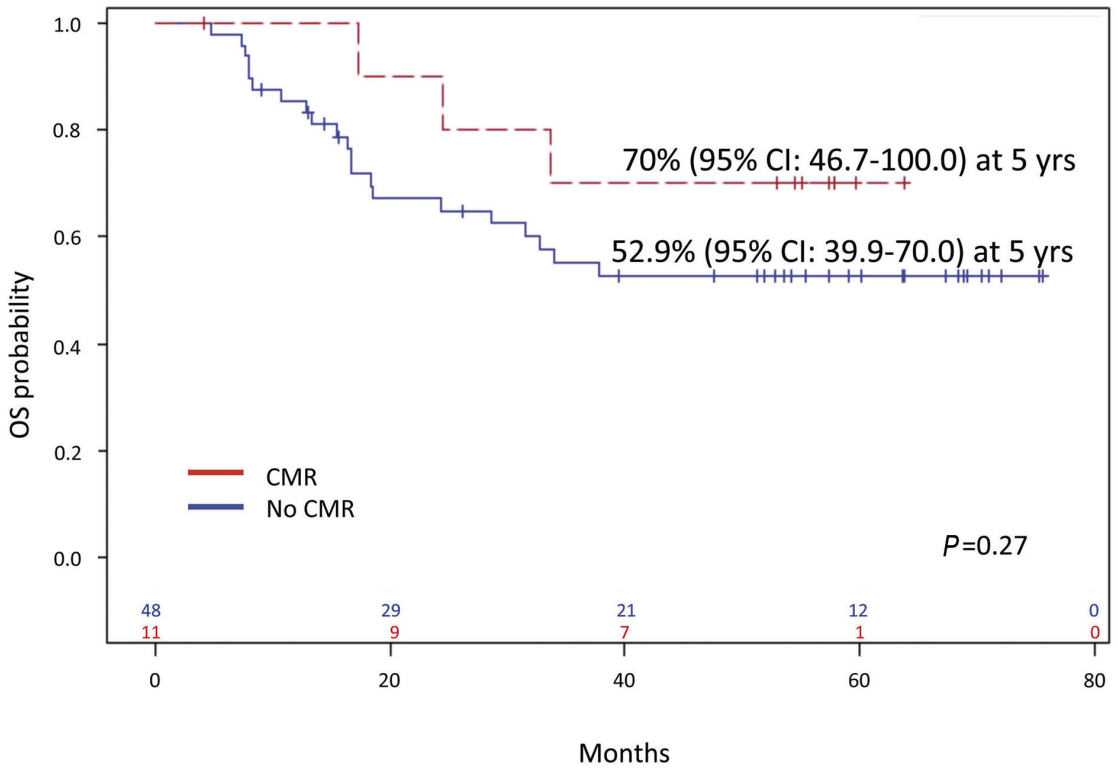

Figure 5. Survival stratified according to molecular response at the end of induction. (A) Disease-free survival (DFS) and (B) overall survival (OS). Cl: Confidence Interval; CHR: complete hematologic remission; yrs: years. mostly in an era in which a plethora of novel compounds are available.

Even if the numbers are small and protocol violations represent a major limit, the DFS of the few cases in persistent CHR and CMR seems to be better than that of patients who did not achieve CMR. This suggest that some patients, regardless of their age, may be spared systemic chemotherapy and transplant procedure. However, the applicability of this strategy must be carefully evaluated and possibly refined by additional genetic information. Indeed, this chemo/transplant-sparing strategy is addressed in an ancillary study (clinicaltrials gov. Identifier: NCT03318770) to our recently published paper on adult $\mathrm{Ph}+\mathrm{ALL}$ patients of all ages treated in induction with dasatinib and consolidated with the bispecific monoclonal antibody blinatumomab. ${ }^{31}$

Regarding patients in CHR who did not achieve CMR, 35 received chemotherapy consolidation with clofarabinecyclophosphamide: overall, this treatment was capable of inducing CMR in 12 cases (34.3\%), thus indicating its effectiveness and in some cases a valuable bridge to transplant. Furthermore, this strategy was safe, since only 18 SAE were recorded in ten patients. Similarly, allo-SCT proved effective in inducing MRD negativity in $32 \%$ of the patients.

Seventeen hematologic relapses (comprising two extramedullary) and 11 deaths in CHR were recorded. We observed a 5 -year DFS of $47.2 \%$ and a 5 -year OS $56.3 \%$, making the outcome of $\mathrm{Ph}+\mathrm{ALL}$ patients almost comparable to that of Ph- ALL cases. These results represent a slight improvement compared to our previous trial - GIMEMA LAL090 $4^{17}$ - based on a chemotherapy-free imatinib administration in induction, followed by consolidation and alloSCT, in which the 5-year DFS and OS were $45.8 \%$ and $48.8 \%$, respectively. The finding that DFS was not significantly superior might be related to the well-established fact that dasatinib, even though more effective in rapidly eradicating a leukemic clone, is not capable of overcoming the occurrence of TKI-resistant mutations, particularly the 


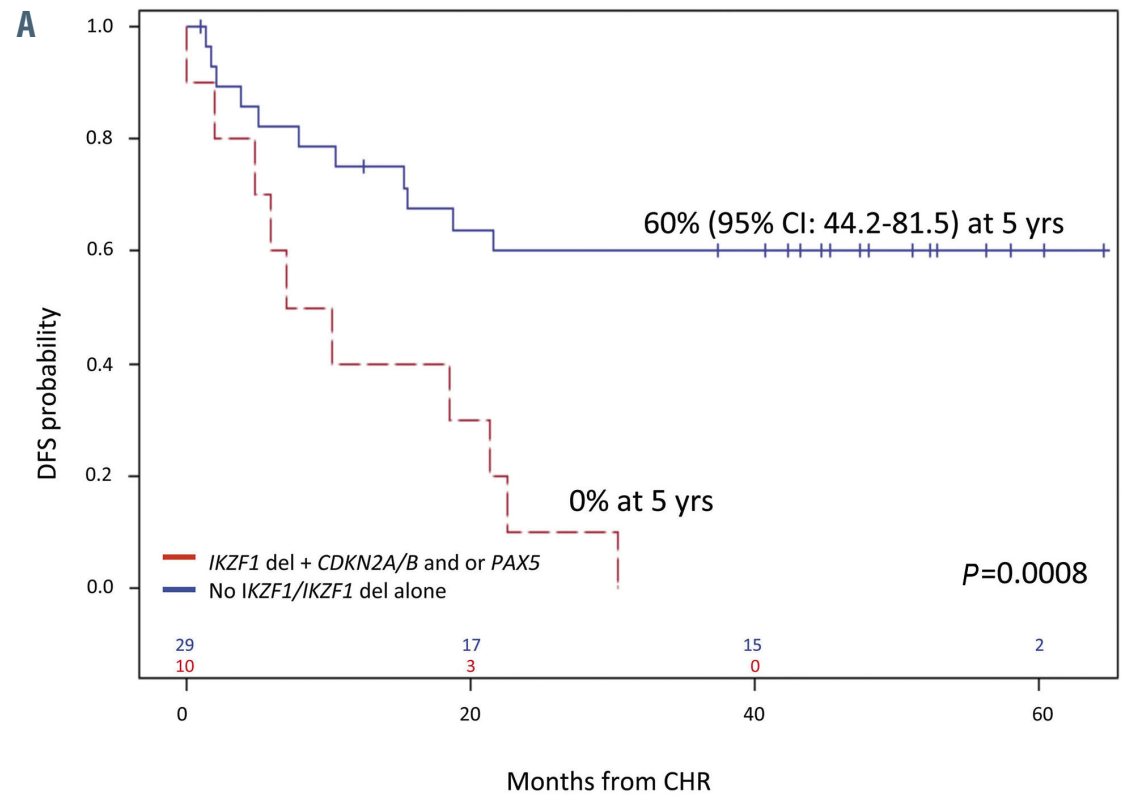

Figure 6. Impact of genetic lesions on survival. (A) Disease-free survival (DFS) and (B) overall survival (OS) of IKZF1 deletion with or without other abnormalities. $\mathrm{Cl}$ : Confidence Interval; CHR: complete hematologic remission; yrs: years.

B

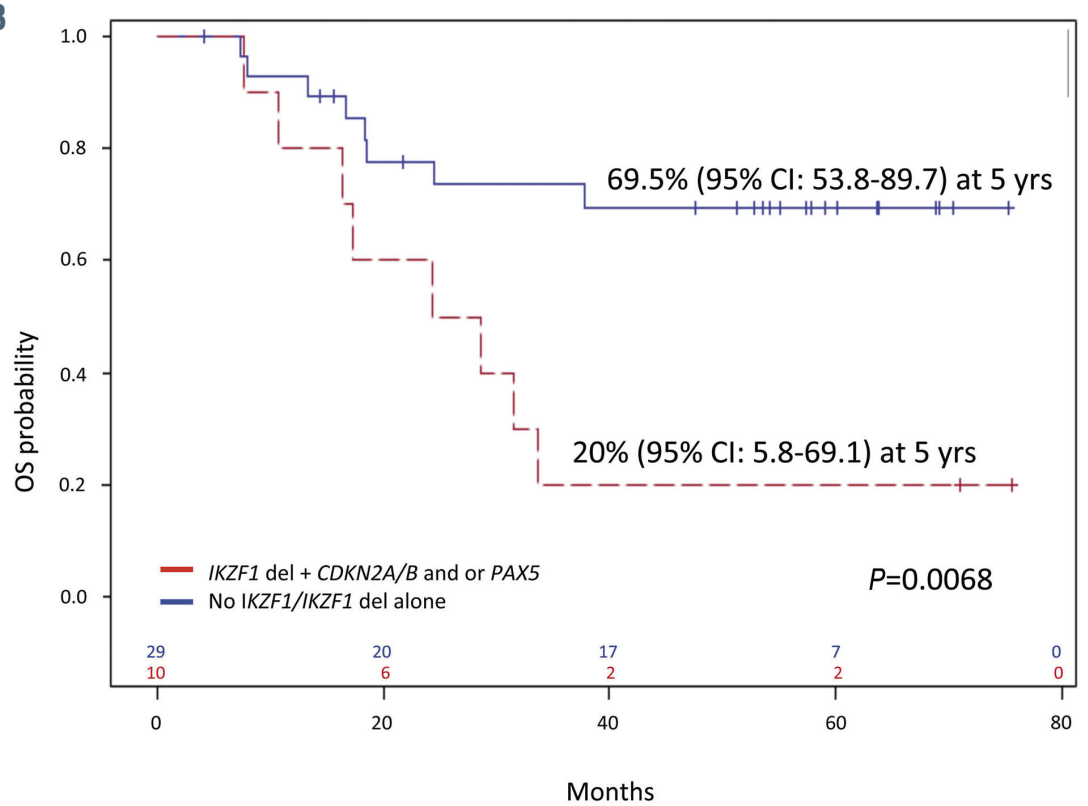

T315I mutation. Although mutations were not systematically screened in this trial, ten patients in hematologic relapse were evaluated and seven indeed harbored a mutation, with T315I being the most frequent $(n=4)$. These results are in line with our previous experience ${ }^{16}$ and with Rousselot and colleagues who reported a high incidence of T315I mutations (18 of 24 relapsing patients, 75\%). ${ }^{32}$ Importantly, three cases were screened and proved positive for $A B L 1$ mutations at MRD increase and none of them relapsed, thus corroborating the notion that an earlier detection can better drive therapeutic decisions. A 7.5\% increase in OS was observed in the current study compared to the results of the LAL0904 protocol, from $48.8 \%$ to $56.3 \%$.

Our results compare favorably also with other studies based on a combination of dasatinib plus chemotherapy, which were also aggravated by toxicities and treatment discontinuations. ${ }^{27,32}$ The European Working Group on Adult ALL used dasatinib in combination with low-dose chemotherapy in elderly patients with Ph+ ALL: 61 patients were enrolled (median age 69 years), 96\% of patients achieved CR after induction, but three patients died. Overall, the 5-year OS was $36 \%$, suggesting that a proportion of patients may experience long-term survival without intensive therapies. ${ }^{32}$ Ravandi et al. ${ }^{27}$ published the final results of their phase II study with dasatinib plus HyperCVAD in 72 adult Ph+ ALL patients: they reported CHR of $96 \%$ with $83 \%$ of complete cytogenetic responses after induction with three early deaths. Overall, the 5-years DFS and OS were $44 \%$ and $46 \%$, respectively. The US Intergroup reported the results of a multicenter trial for $\mathrm{Ph}+$ ALL patients aged 18-60 years treated with dasatinib plus HyperCVAD and allo-SCT for eligible patients. ${ }^{33}$ The overall complete remission/complete remission with incomplete hematologic recovery (CR-CRi) rate was $88 \%$ and the 3 -year OS and DFS were $69 \%$ and $62 \%$, respectively. The shorter follow-up does not allow a direct comparison with our study. In addition, a sizable proportion of patients $(n=34)$ had received one course of therapy prior to enroll- 
ment in the study, including 16 who had already achieved a CR or CRi.

In the pre-TKI era, allo-SCT in first remission was considered the standard of care for all eligible patients. After the introduction of TKI, the use of allo-SCT in first CHR has been questioned. ${ }^{19,3438}$ Although our study was not powered to define its role, allo-SCT did not seem to have an impact on survival. This might be due to the small sample size of patients who performed the procedure and may also be influenced by protocol violations, which represent a major limit of the study: indeed, five patients in CMR after dasatinib induction underwent allo-SCT. Finally, allo-SCT was aggravated by mortality in five patients. In the report by Ravandi et al..$^{27}$ only a small proportion of patients underwent allo-SCT in first remission without any improvement in survival. A recent report from Chang and colleagues ${ }^{36}$ also reported no benefit in OS and DFS at 3 years between transplanted and nontransplanted patients in a retrospective study $(76 \%$ vs. $71.3 \%, P=0.56 ; 70.5 \%$ vs. $80.1 \%, P=0.94$, respectively) in $\mathrm{Ph}+\mathrm{ALL}$ patients treated with dasatinib plus chemotherapy followed or not by allo-SCT. In contrast, in the report by the US Intergroup study, the landmark analysis at 175 days showed a significant advantage in both OS and relapse-free survival $(P=0.038$ and $P=0.0037$, respectively) in transplanted patients..$^{33}$ In our cohort of transplanted patients, for patients relapsed after allo-SCT, all of them were MRD-positive before the procedure. This finding is in line with a recent publication by Candoni and colleagues in which the 5-year CIR was higher in Ph+ ALL patients that were $\mathrm{MRD}+$ prior to the allograft. ${ }^{37}$

With regard to the specific biologic features investigated, we observed, during induction, a significantly better clearance of the BCR-ABL1 transcript in p190 patients as opposed to p210 patients; interestingly, this, however, did not translate into an improved DFS and OS, thus suggesting that the kinetics of MRD clearance is different between the p190 and p210 cases. Further investigations are ongoing on this specific issue. Similarly, DFS was not statically better for patients achieving CMR after induction, mostly because of the small sample size and also indicating that patients who failed to achieve CMR can be rescued by an effective post-induction treatment.

At variance, patients with IKZF1 deletions plus $C D K N 2 A / B$ and/or PAX5 deletions had a significantly worse DFS and OS, all relapsing within 24 months: this finding held true also in cases that achieved CMR. Also, the IKZF1-plus signature was the only prognostic factor to have an impact on DFS and OS in both univariate and multivariate analyses. Therefore, screening for these genetic lesions should be performed in all $\mathrm{Ph}+$ ALL patients at the time of diagnosis for a more refined prognostic stratification, and to further optimize treatment. $23,26,31$

Taken together, the results of this study document that durable remissions and long-term survival can be achieved with a total therapy strategy based on a chemotherapy-free induction, with an OS rate of $56.3 \%$ at a median follow-up of 57.4 months. Even in the TKI era, allo-SCT still remains an effective treatment for eligible adult patients; further efforts need to be made to identify which patients may be spared the procedure and its related toxicity, through the design of trials with a larger sample size that allow patients' stratification according to their molecular features. Whether the use of more powerful BCR-ABL1 inhibitors such as ponatinib, may improve OS in Ph+ ALL remains to be determined. ${ }^{38,39}$ The cardiovascular toxicity of ponatinib may counteract the potential benefit of this drug in patients with serious comorbidities. Finally, the upfront integration of TKI with immunoconjugates or BiTE antibodies - such as blinatumomab - appears capable of inducing a high percentage of molecular response in adult $\mathrm{Ph}+$ ALL patients and could eventually lead to higher rates of long-term responses. ${ }^{31}$

\section{Disclosures}

$R F$ has served on advisory boards and at speaker bureaus of Janssen, AbbVie, Novartis, Amgen, Incyte, Pfizer and Servier; SC has served on advisory boards of Amgen, Pfizer, Incyte and Shire; the other authors declare no conflicts of interest.

\section{Contributions}

SC provided samples and clinical data, analyzed data and wrote the manuscript; MA analyzed data and wrote the manuscript; $A V$ provided clinical data; $L E$ and $M M$ performed experiments; AP performed statistical analyses; $P F$ contributed to protocol management; $F D R, L S, F F, C C, G M, F R, F F, N C, C B, A T, S S$, $N D R, A M$ and GB provided clinical data; $M V$ contributed to protocol management; $R F$ designed the research and the trial, and critically revised the manuscript.

\section{Acknowledgements}

The authors wish to thank Sanofi and Bristol Myers Squibb, which provided clofarabine and dasatinib, respectively, free of charge.

\section{Funding}

The authors wish to thank the Associazione Italiana per la Ricerca sul Cancro (AIRC) 5×1000, Special Program Metastases (21198), Milan (Italy) to RF; Finanziamento Medi Progetti Universitari 2015 to SC (Sapienza University of Rome) and PRIN 2017 (2017PPS2X4_002) to SC.

\section{References}

1. Nowell PC, Hungerford DA. Chromosome studies on normal and leukemic human leukocytes. J Natl Cancer Inst. 1960;25:85109

2. Mancini M, Scappaticci D, Cimino G, et al. A comprehensive genetic classification of adult acute lymphoblastic leukemia (ALL): analysis of the GIMEMA 0496 protocol. Blood. 2005;105(9):3434-3441.

3. Chiaretti S, Vitale A, Cazzaniga G, et al. Clinico-biological features of 5202 patients with acute lymphoblastic leukemia enrolled in the Italian AIEOP and GIMEMA protocols and stratified in age cohorts. Haematologica. 2013;98(11):1702-1710.

4. Burmeister T, Schwartz S, Bartram CR, et al. Patients' age and BCR-ABL frequency in adult B- precursor ALL: a retrospective analysis from the GMALL study group. Blood. 2008;112(3):918-919.

5. Yanada M, Takeuchi J, Sugiura I, et al. High complete remission rate and promising outcome by combination of imatinib and chemotherapy for newly diagnosed BCRABL-positive acute lymphoblastic leukemia: a phase II study by the Japan Adult Leukemia Study Group. J Clin Oncol. 2006;24(3):460-466.
6. Talpaz M, Shah NP, Kantariian $\mathrm{H}$, et al Dasatinib in imatinib-resistant Philadelphia chromosome-positive leukemias. N Engl J Med. 2006;354(24):2531-2541.

7. Vignetti M, Fazi P, Cimino G, et al. Imatinib plus steroids induces complete remissions and prolonged survival in elderly Philadelphia chromosome-positive patients with acute lymphoblastic leukemia without additional chemotherapy: results of the Gruppo Italiano Malattie Ematologiche dell'Adulto (GIMEMA) LAL0201-B protocol. Blood. 2007;109(9):3676-3678.

8. Ottmann OG, Wassmann B, Pfeifer $\mathrm{H}$, et al. Imatinib compared with chemotherapy as 
front-line treatment of elderly patients with Philadelphia chromosome-positive acute lymphoblastic leukemia (Ph+ALL). Cancer. 2007;109(10):2068-2076.

9. Bassan R, Rossi G, Pogliani EM, et al. Chemotherapy-phased imatinib pulses improve long- term outcome of adult patients with Philadelphia chromosomepositive acute lymphoblastic leukemia: Northern Italy Leukemia Group protocol 09/00. J Clin Oncol. 2010;28(22):3644-3652.

10. Dombret H, Gabert J, Boiron JM, et al. Outcome of treatment in adults Philadelphia chromosome-positive acute lymphoblastic leukemia-results of the prospective multi-center LALA-94 trial. Blood. 2002;100(7):2357-2366.

11. Laport GG, Alvarnas JC, Palmer JM. Longterm remission of Philadelphia chromosome-positive acute lymphoblastic leukemia after allogeneic hematopoietic cell transplantation from matched sibling donors: a 20 -year experience with the fractionated total body irradiation-etoposide regimen. Blood. 2008; 112(3):903-909.

12. Fielding AK, Rowe JM, Richards SM. Prospective outcome data on 267 unselected adult patients with Philadelphia chromosome-positive acute lymphoblastic leukemia confirms superiority of allogeneic transplantation over chemotherapy in the pre-imatinib era: results from the International ALL Trial MRC UKALLXII/ECOG2993. Blood. 2009; 113(19):4489-4496

13. Wassmann B, Pfeifer H, Goekbuget N, et al. Alternating versus concurrent schedules of imatinib and chemotherapy as front-line therapy for Philadelphia-positive acute lymphoblastic leukemia (Ph+ALL). Blood. 2006;108(5):1469-1477.

14. de Labarthe A, Rousselot P, Huguet-Rigal F, et al. Group for Research on Adult Acute Lymphoblastic Leukemia (GRAALL). Imatinib combined with induction or consolidation chemotherapy in patients with de novo Philadelphia chromosome-positive acute lymphoblastic leukemia: results of the GRAAPH-2003 study. Blood. 2007;109 (4):1408-1413

15. Ribera JM, Oriol A, Gonzalez M, et al. Concurrent intensive chemotherapy and imatinib before and after stem cell transplantation in newly diagnosed Philadelphia chromosome-positive acute lymphoblastic leukemia. Final results of the CSTIBES02 trial. Haematologica. 2010;95(1):87-95.

16. Foà R, Vitale A, Vignetti M, et al. Dasatinib as first-line treatment for adult patients with Philadelphia chromosome-positive acute lymphoblastic leukemia. Blood. 2011;118(25):6521-6528

17. Chiaretti S, Vitale A, Vignetti M, et al. A sequential approach with imatinib, chemotherapy and transplant for adult $\mathrm{Ph}+$ acute lymphoblastic leukemia: final results of the GIMEMA LAL0904 study. Haematologica. 2016;101(12):1544-1552.

18. Fielding AK, Rowe JM, Buck G, et al.
UKALLXII/ECOG2993: addition of imatinib to a standard treatment regimen enhances long-term outcomes in Philadelphia positive acute lymphoblastic leukemia. Blood. 2014;123(6):843-850.

19. Ribera JM, García O, Montesinos P, et al. Treatment of young patients with Philadelphia chromosome-positive acute lymphoblastic leukaemia using increased dose of imatinib and deintensified chemotherapy before allogeneic stem cell transplantation. Br J Haematol. 2012;159 (1):78-81.

20. Chalandon Y, Thomas X, Hayette S, et al; Group for Research on Adult Acute Lymphoblastic Leukemia (GRAALL). Randomized study of reduced-intensity chemotherapy combined with imatinib in adults with Ph-positive acute lymphoblastic leukemia. Blood. 2015;125(24):37113719.

21. Gabert J, Beillard E, van der Velden VH, et al. Standardization and quality control studies of 'real-time' quantitative reverse transcriptase polymerase chain reaction of fusion gene transcripts for residual disease detection in leukemia - a Europe Against Cancer program. Leukemia. 2003;17(12): 2318-2357.

22. Elia L, Mancini M, Moleti L, et al. A multiplex reverse transcriptase polymerase chain reaction strategy for the diagnostic molecular screening of chimeric genes: a clinical evaluation on 170 patients with acute lymphoblastic leukemia. Haematologica. 2003;88(3):275-279.

23. Fedullo AL, Messina M, Elia L, et al. Prognostic implications of additional genomic lesions in adult Philadelphia chromosomepositive acute lymphoblastic leukemia. Haematologica. 2019;104(2):312-318.

24. Harris PA, Taylor R, Thielke R, et al. Research electronic data capture (REDCap) - A metadata- driven methodology and workflow process for providing translational research informatics support. Biomed Inform. 2009;42(2):377-381.

25. Harris PA, Taylor R, Minor BL, et al. The REDCap Consortium: building an international community of software partners. J Biomed Inform. 2019;95:103208.

26. Pfeifer $\mathrm{H}$, Raum $\mathrm{K}$, Markovic $\mathrm{S}$, et al. Genomic CDKN2A/2B deletions in adult $\mathrm{Ph}+\mathrm{ALL}$ are adverse despite allogeneic stem cell transplantation. Blood. 2018;131 (13):1464-1475

27. Ravandi F, O'Brien SM, Cortes JE, et al. Long-term follow-up of a phase 2 study of chemotherapy plus dasatinib for the initial treatment of patients with Philadelphia chromosome-positive acute lymphoblastic leukemia. Cancer. 2015;121(23):4158-4164.

28. Daver N, Thomas D, Ravandi F, et al. Final report of a phase II study of imatinib mesylate with hyper-CVAD for the front-line treatment of adult patients with Philadelphia chromosome-positive acute lymphoblastic leukemia. Haematologica. 2015;100(5):653-661.
29. Lim SN, Joo YD, Lee KH, et al. Long-term follow-up of imatinib plus combination chemotherapy in patients with newly diagnosed Philadelphia chromosome-positive acute lymphoblastic leukemia. Am J Hematol. 2015;90(11):1013-1020.

30. Hatta Y, Mizuta S, Matsuo $\mathrm{K}$, et al. Final analysis of the JALSG Ph+ALL202 study: tyrosine kinase inhibitor-combined chemotherapy for Ph+ ALL. Ann Hematol. 2018;97(9):1535-1545.

31. Foà R, Bassan R, Vitale A, et al. Dasatinibblinatumomab for Ph-positive acute lymphoblastic leukemia in adults. N Engl J Med. 2020;383(17):1613-1623.

32. Rousselot P, Coudé MM, Gokbuget N, et al. Dasatinib and low-intensity chemotherapy in elderly patients with Philadelphia chromosome-positive ALL. Blood. 2016;128(6): 774-782.

33. Ravandi F, Othus M, O'Brien SM, et al. US Intergroup study of chemotherapy plus dasatinib and allogeneic stem cell transplant in Philadelphia chromosome positive ALL. Blood Adv, 2016;1(3):250-259.

34. Kim DY, Joo YD, Lim SN, et al. Nilotinib combined with multiagent chemotherapy for newly diagnosed Philadelphia-positive acute lymphoblastic leukemia. Blood. 2015;126(6):746-756.

35. Short N, Kantariian HM, Ravandi F, et al Long-term safety and efficacy of HyperCVAD plus ponatinib as frontline therapy for adults with Philadelphia chromosomepositive acute lymphoblastic leukemia. Blood. 2019;134(Suppl. 1):S283.

36. Chang I, Douer D, Aldoss I, et al. Combination chemotherapy plus dasatinib leads to comparable overall survival and relapse-free survival rates as allogeneic hematopoietic stem cell transplantation in Philadelphia positive acute lymphoblastic leukemia. Cancer Med. 2019;8(6):28322839

37. Candoni A, Rambaldi A, Fanin R, et al. Outcome of allogeneic hematopoietic stem cell transplantation in adult patients with Philadelphia chromosome-positive acute lymphoblastic leukemia in the era of tyrosine kinase inhibitors: a registry-based study of the Italian Blood and Marrow Transplantation Society (GITMO). Biol Blood Marrow Transplant. 2019;25(12): 2388-2397.

38. Jabbour E, Short NJ, Ravandi F, et al Combination of hyper-CVAD with ponatinib as first-line therapy for patients with Philadelphia chromosome-positive acute lymphoblastic leukaemia: long-term follow-up of a single-centre, phase 2 study. Lancet Haematol. 2018;5(12):e618-e627.

39. Martinelli G, Piciocchi A, Papayannidis C, et al. First report of the GIMEMA LAL1811 phase II prospective study of the combination of steroids with ponatinib as frontline therapy of elderly or unfit patients with Philadelphia chromosome-positive acute lymphoblastic leukemia. Blood. 2017;130 (Suppl.1):S99. 